\title{
TWO BIFURCATION SETS ARISING FROM THE BETA TRANSFORMATION WITH A HOLE AT 0
}

\author{
SIMON BAKER AND DERONG KONG
}

\begin{abstract}
Given $\beta \in(1,2]$, the $\beta$-transformation $T_{\beta}: x \mapsto \beta x(\bmod 1)$ on the circle $[0,1)$ with a hole $[0, t)$ was investigated by Kalle et al. (2019). They described the set-valued bifurcation set

$$
\mathscr{E}_{\beta}:=\left\{t \in[0,1): K_{\beta}\left(t^{\prime}\right) \neq K_{\beta}(t) \forall t^{\prime}>t\right\},
$$

where $K_{\beta}(t):=\left\{x \in[0,1): T_{\beta}^{n}(x) \geq t \forall n \geq 0\right\}$ is the survivor set. In this paper we investigate the dimension bifurcation set

$$
\mathscr{B}_{\beta}:=\left\{t \in[0,1): \operatorname{dim}_{H} K_{\beta}\left(t^{\prime}\right) \neq \operatorname{dim}_{H} K_{\beta}(t) \forall t^{\prime}>t\right\},
$$

where $\operatorname{dim}_{H}$ denotes the Hausdorff dimension. We show that if $\beta \in(1,2]$ is a multinacci number then the two bifurcation sets $\mathscr{B}_{\beta}$ and $\mathscr{E}_{\beta}$ coincide. Moreover we give a complete characterization of these two sets. As a corollary of our main result we prove that for $\beta$ a multinacci number we have $\operatorname{dim}_{H}\left(\mathscr{E}_{\beta} \cap[t, 1]\right)=\operatorname{dim}_{H} K_{\beta}(t)$ for any $t \in[0,1)$. This confirms a conjecture of Kalle et al. for $\beta$ a multinacci number.
\end{abstract}

\section{INTRODUCTION}

Given $\beta \in(1,2]$, the $\beta$-transformation $T_{\beta}$ on the circle $\mathbb{R} / \mathbb{Z} \sim[0,1)$ is defined by

$$
T_{\beta}:[0,1) \rightarrow[0,1) ; \quad x \mapsto \beta x \quad(\bmod 1) .
$$

Following the pioneering work of Rényi [9] and Parry [7] there has been a great interest in the study of $T_{\beta}$. In general, the system $\Phi_{\beta}=\left([0,1), T_{\beta}\right)$ does not admit a Markov partition, this makes describing the dynamics of $\Phi_{\beta}$ more challenging.

When $\beta=2$, Urbański [11, 12] considered the open dynamical system under the doubling map $T_{2}$ with a hole at zero. More precisely, for $t \in[0,1)$ let

$$
K_{2}(t):=\left\{x \in[0,1): T_{2}^{n}(x) \geq t \forall n \geq 0\right\} .
$$

He showed that the dimension function $t \mapsto \eta_{2}(t):=\operatorname{dim}_{H} K_{2}(t)$ is a Devil's staircase on $[0,1)$, in particular $\eta_{2}$ satisfies the following properties: (i) $\eta_{2}$ is decreasing and continuous on $[0,1)$; (ii) $\eta_{2}$ is locally constant almost everywhere on $\left[0,1\right.$ ); and (iii) $\eta_{2}$ is not constant on $[0,1)$. Here and throughout the paper $\operatorname{dim}_{H}$ denotes the Hausdorff dimension. Moreover, he investigated the bifurcation sets

$$
\mathscr{E}_{2}:=\left\{t \in[0,1): K_{2}\left(t^{\prime}\right) \neq K_{2}(t) \forall t^{\prime}>t\right\}, \quad \mathscr{B}_{2}:=\left\{t \in[0,1): \eta_{2}\left(t^{\prime}\right) \neq \eta_{2}(t) \forall t^{\prime}>t\right\} .
$$

Clearly, $\mathscr{B}_{2} \subseteq \mathscr{E}_{2}$. In [11] Urbański showed that $\mathscr{B}_{2}=\mathscr{E}_{2}$, and its topological closure $\overline{\mathscr{B}_{2}}$ is a Cantor set, i.e., a non-empty compact set that has neither isolated nor interior points. Furthermore, the following local dimension property was shown to hold: $\lim _{r \rightarrow 0} \operatorname{dim}_{H}\left(\mathscr{E}_{2} \cap\right.$

Date: 16th April 2019.

2010 Mathematics Subject Classification. Primary: 37B10, Secondary: 28A78, 11A63.

Key words and phrases. Bifurcation sets; beta transformation; local dimension; survivor set. 
$(t-r, t+r))=\eta_{2}(t)$ for all $t \in \mathscr{E}_{2}$. Recently, Carminati and Tiozzo in [1] showed that the local Hölder exponent of the dimension function $\eta_{2}$ at any $t \in \mathscr{E}_{2}$ equals $\eta_{2}(t)$.

Inspired by the work of Urbański [11, 12, Kalle et al. in 4 considered the analogous problem for the $\beta$-transformation with a hole $[0, t)$. More precisely, for $t \in[0,1)$ they investigated the survivor set

$$
K_{\beta}(t):=\left\{x \in[0,1): T_{\beta}^{n}(x) \geq t \forall n \geq 0\right\},
$$

and showed that the dimension function $t \mapsto \operatorname{dim}_{H} K_{\beta}(t)$ is also a Devil's staircase on [0,1). Furthermore, they characterized the set-valued bifurcation set

$$
\mathscr{E}_{\beta}:=\left\{t \in[0,1): K_{\beta}\left(t^{\prime}\right) \neq K_{\beta}(t) \forall t^{\prime}>t\right\},
$$

and proved that $\mathscr{E}_{\beta}$ is a Lebesgue null set of full Hausdorff dimension for any $\beta \in(1,2)$. Interestingly, they showed that $\mathscr{E}_{\beta}$ contains infinitely many isolated points for Lebesgue almost every $\beta \in(1,2)$. This is in contrast to the case where $\beta=2$ and $\mathscr{E}_{2}$ has no isolated points.

Since for each $\beta \in(1,2)$ the dimension function $\eta_{\beta}: t \mapsto \operatorname{dim}_{H} K_{\beta}(t)$ is a Devil's staircase, it is natural to consider the dimension bifurcation set

$$
\mathscr{B}_{\beta}:=\left\{t \in[0,1): \eta_{\beta}\left(t^{\prime}\right) \neq \eta_{\beta}(t) \forall t^{\prime}>t\right\} .
$$

This set records those $t$ for which the dimension function $\eta_{\beta}$ has a 'change' within any right neighborhood. Since $\eta_{\beta}$ is continuous, $\mathscr{B}_{\beta}$ cannot have isolated points. On the other hand, the set-valued bifurcation set $\mathscr{E}_{\beta}$ contains (infinitely many) isolated points for Lebesgue almost every $\beta \in(1,2)$. So in general we cannot expect the coincidence of the two bifurcation sets $\mathscr{B}_{\beta}$ and $\mathscr{E}_{\beta}$. That being said, in this paper we show that if $\beta$ is a multinacci number, i.e., the unique root in $(1,2)$ of the equation

$$
x^{m+1}=x^{m}+x^{m-1}+\cdots+x+1
$$

for some $m \in \mathbb{N}$, then the two bifurcation sets indeed coincide.

When $\beta \in(1,2)$ is a multinacci number, the following result for the set-valued bifurcation set $\mathscr{E}_{\beta}$ was established in [4, Theorems $\mathrm{C}$ and D]. We record it here for later use.

Theorem 1.1 ([4]). Let $\beta \in(1,2]$ be a multinacci number. Then the topological closure $\overline{\mathscr{E}_{\beta}}$ is a Cantor set. Furthermore, $\max \overline{\mathscr{E}_{\beta}}=1-1 / \beta$.

In order to give a complete description of the dimension bifurcation set $\mathscr{B}_{\beta}$ we introduce a class of basic intervals.

Definition 1.2. Let $\beta \in(1,2]$. A word $s_{1} \ldots s_{m}$ is called $\beta$-Lyndon if

$$
s_{i+1} \ldots s_{m} \succ s_{1} \ldots s_{m-i} \quad \forall 1 \leq i<m, \quad \text { and } \quad \sigma^{n}\left(\left(s_{1} \ldots s_{m}\right)^{\infty}\right) \prec \delta(\beta) \quad \forall n \geq 0 .
$$

Accordingly, an interval $\left[t_{L}, t_{R}\right) \subset[0,1)$ is called a $\beta$-Lyndon interval if there exists a $\beta$ Lyndon word $s_{1} \ldots s_{m}$ such that

$$
t_{L}=\sum_{i=1}^{m} \frac{s_{i}}{\beta^{i}} \quad \text { and } \quad t_{R}=\frac{\beta^{m}}{\beta^{m}-1} \cdot t_{L} .
$$

In the above definition $\succ$ corresponds to the usual lexicographic ordering and $\delta(\beta)$ is the quasi-greedy $\beta$-expansion of 1 . These are both defined formally in the next section.

We will show that the $\beta$-Lyndon intervals are pairwise disjoint for all $\beta \in(1,2]$, and when $\beta$ is multinacci they cover the interval $[0,1-1 / \beta)$ up to a Lebesgue null set. The latter 
statement can be seen as a consequence of our main result for the coincidence of the two bifurcation sets, which we state below.

Theorem 1. Let $\beta \in(1,2]$ be a multinacci number. Then

$$
\begin{aligned}
\mathscr{B}_{\beta}=\mathscr{E}_{\beta} & =\left[0,1-\frac{1}{\beta}\right) \backslash \bigcup\left[t_{L}, t_{R}\right) \\
& =\left\{t \in[0,1): \lim _{r \rightarrow 0} \operatorname{dim}_{H}\left(\mathscr{B}_{\beta} \cap(t, t+r)\right)=\operatorname{dim}_{H} K_{\beta}(t)>0\right\},
\end{aligned}
$$

where the union is taken over all pairwise disjoint $\beta$-Lyndon intervals.

By Theorem 1 it follows that the topological closure $\left[t_{L}, t_{R}\right]$ of each $\beta$-Lyndon interval is indeed a maximal interval where the dimension function $\eta_{\beta}$ is constant. As a corollary of Theorem 1] we confirm a conjecture of [4] for $\beta$ a multinacci number.

Corollary 2. If $\beta \in(1,2]$ is a multinacci number, then

$$
\operatorname{dim}_{H}\left(\mathscr{E}_{\beta} \cap[t, 1]\right)=\operatorname{dim}_{H} K_{\beta}(t) \quad \forall t \in[0,1) .
$$

The rest of the paper is organized as follows. In the next section we recall some properties from symbolic dynamics and the dimension formula for the survivor set $K_{\beta}(t)$. The proof of Theorem 1 and Corollary 2 will be given in Section 3 , In the final section we make some remarks and point out that the method of proof for Theorem 1 can be applied to some other special values of $\beta$.

\section{Preliminaries and $\beta$-Lyndon Intervals}

Given $\beta \in(1,2]$, for each $x \in I_{\beta}:=[0,1 /(\beta-1)]$ there exists a sequence $\left(d_{i}\right)=d_{1} d_{2} \ldots \in$ $\{0,1\}^{\mathbb{N}}$ such that

$$
x=\sum_{i=1}^{\infty} \frac{d_{i}}{\beta^{i}}=:\left(\left(d_{i}\right)\right)_{\beta} .
$$

The sequence $\left(d_{i}\right)$ is called a $\beta$-expansion of $x$. Sidorov [10] showed that for $\beta \in(1,2)$ Lebesgue almost every $x \in I_{\beta}$ has a continuum of $\beta$-expansions. This is rather different from the case when $\beta=2$ where every number in $I_{2}=[0,1]$ has a unique dyadic expansion except for countably many points that have precisely two expansions. Given $x \in I_{\beta}$, among all of its $\beta$-expansions let $b(x, \beta)=\left(b_{i}(x, \beta)\right)$ be the greedy $\beta$-expansion of $x$, i.e., the lexicographically largest $\beta$-expansion of $x$. Such a sequence always exists and is generated by the orbit of $x$ under the map $T_{\beta}$. Similarly, for $x \in(0,1 /(\beta-1)]$ let $a(x, \beta)=\left(a_{i}(x, \beta)\right)$ be the quasi-greedy $\beta$-expansion of $x$ (cf. [2]), which is the lexicographically largest $\beta$-expansion of $x$ not ending with $0^{\infty}$. Here for a word $\mathbf{c}$ we denote by $\mathbf{c}^{\infty}:=\mathbf{c c} \cdots$ the periodic sequence with periodic block c. Throughout the paper we will use the lexicographic order between sequences and words in the usual way. For example, for two sequences $\left(c_{i}\right),\left(d_{i}\right) \in\{0,1\}^{\mathbb{N}}$ we write $\left(c_{i}\right) \prec\left(d_{i}\right)$ if $c_{1}<d_{1}$, or there exists $n>1$ such that $c_{1} \ldots c_{n-1}=d_{1} \ldots d_{n-1}$ and $c_{n}<d_{n}$. Furthermore, for two words $\mathbf{c}$, $\mathbf{d}$ we say $\mathbf{c} \prec \mathbf{d}$ if $\mathbf{c} 0^{\infty} \prec \mathbf{d} 0^{\infty}$.

For $\beta \in(1,2]$ let

$$
\delta(\beta)=\delta_{1}(\beta) \delta_{2}(\beta) \ldots
$$


be the quasi-greedy $\beta$-expansion of 1 , i.e., $\delta(\beta)=a(1, \beta)$. Let $\sigma$ be the left-shift on $\{0,1\}^{\mathbb{N}}$ defined by $\sigma\left(\left(c_{i}\right)\right)=\left(c_{i+1}\right)$. Then $b\left(T_{\beta}(x), \beta\right)=\sigma(b(x, \beta))$ for any $x \in[0,1)$. The following lexicographic characterizations of $\delta(\beta)$ and the greedy expansion $b(x, \beta)$ are essentially due to Parry [7] (see also [3]).

Lemma 2.1. (i) The map $\beta \mapsto \delta(\beta)$ is a strictly increasing bijection from $(1,2]$ onto the set of sequences $\left(\delta_{i}\right) \in\{0,1\}^{\mathbb{N}}$ not ending with $0^{\infty}$ and satisfying $\sigma^{n}\left(\left(\delta_{i}\right)\right) \preccurlyeq\left(\delta_{i}\right) \forall n \geq 0$.

(ii) Let $\beta \in(1,2]$. Then the map $x \mapsto b(x, \beta)$ is a strictly increasing bijection from $[0,1)$ onto the set of all sequences $\left(b_{i}\right) \in\{0,1\}^{\mathbb{N}}$ satisfying $\sigma^{n}\left(\left(b_{i}\right)\right) \prec \delta(\beta) \forall n \geq 0$.

(iii) For any $\beta \in(1,2)$ the sequence $b(1, \beta)=\left(b_{i}\right)$ satisfies $\sigma^{n}\left(\left(b_{i}\right)\right) \prec \delta(\beta) \forall n \geq 1$.

For $\beta \in(1,2]$ let $\left[t_{L}, t_{R}\right)$ be a $\beta$-Lyndon interval generated by a $\beta$-Lyndon word $s_{1} \ldots s_{m}$. Then by Definition 1.2 and Lemma 2.1 (ii) it follows that

$$
b\left(t_{L}, \beta\right)=s_{1} \ldots s_{m} 0^{\infty} \text { and } b\left(t_{R}, \beta\right)=\left(s_{1} \ldots s_{m}\right)^{\infty} .
$$

Lemma 2.2. For any $\beta \in(1,2]$ the $\beta$-Lyndon intervals are pairwise disjoint.

Proof. Let $\left[t_{L}, t_{R}\right)$ and $\left[t_{L}^{\prime}, t_{R}^{\prime}\right)$ be two $\beta$-Lyndon intervals generated by the $\beta$-Lyndon words $s_{1} \ldots s_{p}$ and $s_{1}^{\prime} \ldots s_{q}^{\prime}$, respectively. Suppose on the contrary that $\left[t_{L}, t_{R}\right) \cap\left[t_{L}^{\prime}, t_{R}^{\prime}\right) \neq \emptyset$. Without loss of generality we assume $t_{L}<t_{L}^{\prime}<t_{R}$. Then by Definition [1.2 and Lemma 2.1(ii) it follows that

$$
s_{1} \ldots s_{p} 0^{\infty} \prec s_{1}^{\prime} \ldots s_{q}^{\prime} 0^{\infty} \prec\left(s_{1} \ldots s_{p}\right)^{\infty} .
$$

This implies $q>p, s_{1}^{\prime} \ldots s_{p}^{\prime}=s_{1} \ldots s_{p}$ and $s_{p+1}^{\prime} \ldots s_{q}^{\prime} 0^{\infty} \prec\left(s_{1} \ldots s_{p}\right)^{\infty}$. Write $q=N p+r$ with $N \geq 1$ and $0<r \leq p$. So, either there exists $1 \leq k<N$ such that

$$
s_{p+1}^{\prime} \ldots s_{k p}^{\prime}=\left(s_{1} \ldots s_{p}\right)^{k-1} \quad \text { and } \quad s_{k p+1}^{\prime} \ldots s_{(k+1) p}^{\prime} \prec s_{1} \ldots s_{p}
$$

or

$$
s_{p+1}^{\prime} \ldots s_{N p}^{\prime}=\left(s_{1} \ldots s_{p}\right)^{N-1} \text { and } s_{N p+1}^{\prime} \ldots s_{q}^{\prime} \preccurlyeq s_{1} \ldots s_{q-N p} .
$$

Using $s_{1}^{\prime} \ldots s_{p}^{\prime}=s_{1} \ldots s_{p}$ we conclude in both cases that

$$
s_{j+1}^{\prime} \ldots s_{q}^{\prime} \preccurlyeq s_{1}^{\prime} \ldots s_{q-j}^{\prime} \quad \text { for some } j \in\{p, p+1, \ldots, q-1\} .
$$

This is not possible by the definition of a $\beta$-Lyndon word.

To describe the Hausdorff dimension of the survivor set

$$
K_{\beta}(t)=\left\{x \in[0,1): T_{\beta}^{n}(x) \geq t \forall n \geq 0\right\},
$$

we recall from [5, Chapter 4] the definition of topological entropy for a symbolic set. For a set $X \subset\{0,1\}^{\mathbb{N}}$, its topological entropy is defined to be

$$
h(X)=\liminf _{n \rightarrow \infty} \frac{\log \# B_{n}(X)}{n},
$$

where $B_{n}(X)$ is the set of all length $n$ prefixes of sequences from $X$.

The following characterization of the set-valued bifurcation set $\mathscr{E}_{\beta}$ was implicitly given in [11] (see also [4, Proposition 2.3]). Furthermore, the Hausdorff dimension of $K_{\beta}(t)$ was implicitly given by Raith in [8], and was recently explicitly presented in [4, Equation (2.6)].

Proposition 2.3. $\quad$ (i) Let $\beta \in(1,2]$. Then

$$
\mathscr{E}_{\beta}=\left\{t \in[0,1): T_{\beta}^{n}(t) \geq t \forall n \geq 0\right\} .
$$


(ii) Let $\beta \in(1,2]$ and $t \in[0,1)$. Then the Hausdorff dimension of $K_{\beta}(t)$ is given by

$$
\operatorname{dim}_{H} K_{\beta}(t)=\frac{h\left(\widetilde{K}_{\beta}(t)\right)}{\log \beta},
$$

where $\widetilde{K}_{\beta}(t):=\left\{\left(x_{i}\right) \in\{0,1\}^{\mathbb{N}}: b(t, \beta) \preccurlyeq \sigma^{n}\left(\left(x_{i}\right)\right) \preccurlyeq \delta(\beta) \forall n \geq 0\right\}$. Furthermore, the dimension function $\eta_{\beta}: t \mapsto \operatorname{dim}_{H} K_{\beta}(t)$ is a Devil's staircase, i.e., $\eta_{\beta}$ is a non-constant, decreasing and continuous function which is locally constant almost everywhere in $[0,1)$.

\section{Proof of Theorem 1}

In this section we will prove Theorem 1. First we show that the dimension bifurcation set $\mathscr{B}_{\beta}$ coincides with the set-valued bifurcation set $\mathscr{E}_{\beta}$, we then derive a complete characterization of these sets via the $\beta$-Lyndon intervals. The proof heavily relies upon the transitivity of the symbolic survivor set $\widetilde{K}_{\beta}(t)$ (see Lemma 3.2 below).

Proposition 3.1. Let $\beta \in(1,2)$ be a multinacci number. Then

$$
\mathscr{B}_{\beta}=\mathscr{E}_{\beta}=\left[0,1-\frac{1}{\beta}\right) \backslash \bigcup\left[t_{L}, t_{R}\right)
$$

where the union is taken over all $\beta$-Lyndon intervals.

Observe by Lemma 2.2 that the $\beta$-Lyndon intervals are pairwise disjoint. In fact the closed $\beta$-Lyndon intervals $\left\{\left[t_{L}, t_{R}\right]\right\}$ are also pairwise disjoint. So by Proposition 3.1 it follows that each closed $\beta$-Lyndon interval is a maximal interval where the dimension function $\eta_{\beta}$ is constant.

The proof of Proposition 3.1 will be split into several lemmas. We fix a multinacci number $\beta \in(1,2)$ with $\delta(\beta)=\left(1^{m} 0\right)^{\infty}$ for some $m \geq 1$. In view of Proposition 2.3 it is necessary to investigate the symbolic survivor set

$$
\widetilde{K}_{\beta}(t)=\left\{\left(x_{i}\right) \in\{0,1\}^{\mathbb{N}}: b(t, \beta) \preccurlyeq \sigma^{n}\left(\left(x_{i}\right)\right) \preccurlyeq \delta(\beta) \forall n \geq 0\right\} .
$$

Lemma 3.2. Let $\beta \in(1,2)$ with $\delta(\beta)=\left(1^{m} 0\right)^{\infty}$, and let $\left[t_{L}, t_{R}\right) \subset[0,1-1 / \beta)$ be a $\beta$-Lyndon interval. Then the set-valued map $t \mapsto \widetilde{K}_{\beta}(t)$ is constant on $\left[t_{L}, t_{R}\right]$, and the set $\widetilde{K}_{\beta}\left(t_{R}\right)$ is a transitive subshift of finite type.

Proof. Suppose $\left[t_{L}, t_{R}\right)$ is a $\beta$-Lyndon interval generated by $s_{1} \ldots s_{p}$. Take $t \in\left[t_{L}, t_{R}\right]$. Then by Lemma 2.1(ii) it follows that

$$
\widetilde{K}_{\beta}\left(t_{R}\right) \subseteq \widetilde{K}_{\beta}(t) \subseteq \widetilde{K}_{\beta}\left(t_{L}\right) .
$$

Observe that $\delta(\beta)=\left(1^{m} 0\right)^{\infty}$ for some $m \in \mathbb{N}$. Then

$$
\begin{aligned}
\widetilde{K}_{\beta}\left(t_{L}\right) & =\left\{\left(x_{i}\right): s_{1} \ldots s_{p} 0^{\infty} \preccurlyeq \sigma^{n}\left(\left(x_{i}\right)\right) \preccurlyeq\left(1^{m} 0\right)^{\infty} \forall n \geq 0\right\} \\
& =\left\{\left(x_{i}\right):\left(s_{1} \ldots s_{p}\right)^{\infty} \preccurlyeq \sigma^{n}\left(\left(x_{i}\right)\right) \preccurlyeq\left(1^{m} 0\right)^{\infty} \forall n \geq 0\right\}=\widetilde{K}_{\beta}\left(t_{R}\right) .
\end{aligned}
$$

Here we use the simple argument that

$$
\sigma^{n}\left(\left(x_{i}\right)\right) \succcurlyeq s_{1} \ldots s_{p} 0^{\infty} \forall n \geq 0 \quad \Longleftrightarrow \quad \sigma^{n}\left(\left(x_{i}\right)\right) \succcurlyeq\left(s_{1} \ldots s_{p}\right)^{\infty} \forall n \geq 0 .
$$


So, the set-valued map $t \mapsto \widetilde{K}_{\beta}(t)$ is constant on $\left[t_{L}, t_{R}\right]$. Furthermore, $\widetilde{K}_{\beta}\left(t_{R}\right)$ is a subshift of finite type with forbidden blocks $c_{1} \ldots c_{k}$ satisfying $c_{1} \ldots c_{k} 0^{\infty} \prec s_{1} \ldots s_{p} 0^{\infty}$ or $c_{1} \ldots c_{k} 0^{\infty} \succ$ $\left(1^{m} 0\right)^{\infty}$, where $k=\max \{p, m+1\}$. It remains to prove the transitivity of $\widetilde{K}_{\beta}\left(t_{R}\right)$.

Since $\left[t_{L}, t_{R}\right) \subset\left[0,1-\frac{1}{\beta}\right)$, by Lemma 2.1 (ii) it follows that $b\left(t_{R}, \beta\right) \prec b\left(1-\frac{1}{\beta}, \beta\right)$, which gives

$$
\left(s_{1} \ldots s_{p}\right)^{\infty} \prec 01^{m} 0^{\infty} .
$$

Arbitrarily fix an admissible word $\varepsilon=\varepsilon_{1} \ldots \varepsilon_{k}$ and an admissible sequence $\gamma=\gamma_{1} \gamma_{2} \ldots$ in $\widetilde{K}_{\beta}\left(t_{R}\right)$. We will construct a word $\nu$ such that $\varepsilon \nu \gamma \in \widetilde{K}_{\beta}\left(t_{R}\right)$. Observe that $\sigma^{n}\left(\left(s_{1} \ldots s_{p}\right)^{\infty}\right) \prec$ $\left(1^{m} 0\right)^{\infty}$ for all $n \geq 0$. Thus, there exists a large integer $N$ such that

$$
\sigma^{n}\left(\left(s_{1} \ldots s_{p}\right)^{\infty}\right) \prec\left(1^{m} 0\right)^{N} 0^{\infty} \quad \text { for all } n \geq 0 .
$$

Denote by $\left(\delta_{i}\right):=\delta(\beta)=\left(1^{m} 0\right)^{\infty}$. Note that $\varepsilon_{i+1} \ldots \varepsilon_{k} \preccurlyeq \delta_{1} \ldots \delta_{k-i}$ for all $0 \leq i<k$. Let $i_{0} \in\{0,1, \ldots, k-1\}$ be the smallest index such that $\varepsilon_{i_{0}+1} \ldots \varepsilon_{k}=\delta_{1} \ldots \delta_{k-i_{0}}$. If such an index $i_{0}$ does not exist, then we put $i_{0}=k$. In either case there exists a word $\mu$ such that $\varepsilon \mu=\varepsilon_{1} \ldots \varepsilon_{i_{0}}\left(1^{m} 0\right)^{N}$. Since $\gamma \preccurlyeq\left(1^{m} 0\right)^{\infty}$, there exists $q \in\{0,1, \ldots, m\}$ such that $\gamma$ begins with $\gamma_{1} \ldots \gamma_{q+1}=1^{q} 0$. We claim that $\varepsilon \mu 1^{m-q} \gamma=\varepsilon_{1} \ldots \varepsilon_{i_{0}}\left(1^{m} 0\right)^{N+1} \gamma_{q+2} \gamma_{q+3} \ldots \in \widetilde{K}_{\beta}\left(t_{R}\right)$, or equivalently,

$$
\left(s_{1} \ldots s_{p}\right)^{\infty} \preccurlyeq \sigma^{n}\left(\varepsilon \mu 1^{m-q} \gamma\right) \preccurlyeq\left(1^{m} 0\right)^{\infty} \quad \text { for all } n \geq 0 .
$$

First we prove the second inequality in (3.4). By the definition of $i_{0}$ it follows that $\sigma^{n}\left(\varepsilon \mu 1^{m-q} \gamma\right) \prec \delta(\beta)=\left(1^{m} 0\right)^{\infty}$ holds for all $0 \leq n<i_{0}$. Furthermore, since $\gamma \in \widetilde{K}_{\beta}\left(t_{R}\right)$, the second inequality in (3.4) also holds for $n \geq|\varepsilon|+|\mu|+m-q$. For the remaining $n$ we observe that $\sigma^{i_{0}}\left(\varepsilon \mu 1^{m-q} \gamma\right)=\left(1^{m} 0\right)^{N+1} \gamma_{q+2} \gamma_{q+3} \ldots$ and $\gamma_{q+2} \gamma_{q+3} \ldots \in \widetilde{K}_{\beta}\left(t_{R}\right)$. So $\sigma^{n}\left(\varepsilon \mu 1^{m-q} \gamma\right) \preccurlyeq\left(1^{m} 0\right)^{\infty}$ for all $i_{0} \leq n<|\varepsilon|+|\mu|+m-q$. This proves the second inequality in (3.4).

For the first inequality in (3.4) we observe that $\varepsilon \mu 1^{m-q} \gamma=\varepsilon_{1} \ldots \varepsilon_{i_{0}}\left(1^{m} 0\right)^{N} 1^{m} \gamma_{q+1} \gamma_{q+2} \ldots$ and $\gamma_{q+1} \gamma_{q+2} \ldots \in \widetilde{K}_{\beta}\left(t_{R}\right)$. Then by (3.2) it follows that $\sigma^{n}\left(\varepsilon \mu 1^{m-q} \gamma\right) \succcurlyeq\left(s_{1} \ldots s_{p}\right)^{\infty}$ for all $n \geq i_{0}$. If $i_{0}=0$, then we are done. Otherwise, we take $0 \leq n<i_{0}$. Since $\varepsilon_{1} \ldots \varepsilon_{i_{0}}$ is an admissible word in $\widetilde{K}_{\beta}\left(t_{R}\right)$, we have

$$
\varepsilon_{n+1} \ldots \varepsilon_{i_{0}} \succcurlyeq t_{1} \ldots t_{i_{0}-n}
$$

where $\left(t_{i}\right):=\left(s_{1} \ldots s_{p}\right)^{\infty}$. The first inequality in (3.4) now holds by (3.3), which tells us that

$$
\left(1^{m} 0\right)^{N} 1^{m} \gamma_{q+1} \gamma_{q+2} \ldots \succ t_{i_{0}-n+1} t_{i_{0}-n+2} \ldots
$$

This completes the proof of our claim.

Since $\varepsilon$ and $\gamma$ are chosen arbitrarily, it follows that $\widetilde{K}_{\beta}\left(t_{R}\right)$ is transitive.

To prove the coincidence of $\mathscr{B}_{\beta}$ and $\mathscr{E}_{\beta}$ we still need the following inequalities.

Lemma 3.3. Let $\left(t_{1} \ldots t_{N}\right)^{\infty} \in\{0,1\}^{\mathbb{N}}$ be a periodic sequence with period $N \geq 2$. If

$$
\sigma^{n}\left(\left(t_{1} \ldots t_{N}\right)^{\infty}\right) \succcurlyeq\left(t_{1} \ldots t_{N}\right)^{\infty} \quad \forall n \geq 0,
$$

then

$$
t_{j+1} \ldots t_{N} \succ t_{1} \ldots t_{N-j} \quad \forall 1 \leq j<N
$$


Proof. Note that $N \geq 2$ is the period of $\left(t_{1} \ldots t_{N}\right)^{\infty}$, and

$$
\sigma^{n}\left(\left(t_{1} \ldots t_{N}\right)^{\infty}\right) \succcurlyeq\left(t_{1} \ldots t_{N}\right)^{\infty} \quad \forall n \geq 0 .
$$

Then $t_{1}=0$ and $t_{N}=1$. Taking the reflection on both sides of (3.5) it follows that

$$
\sigma^{n}\left(\left(\overline{t_{1} \ldots t_{N}}\right)^{\infty}\right) \preccurlyeq\left(\overline{t_{1} \ldots t_{N}}\right)^{\infty} \text { for all } n \geq 0 .
$$

Here for a word $c_{1} \ldots c_{k} \in\{0,1\}^{k}$ its reflection is defined by $\overline{c_{1} \ldots c_{k}}:=\left(1-c_{1}\right)\left(1-c_{2}\right) \ldots(1-$ $\left.c_{k}\right)$. By Lemma 2.1(i) it follows that $\left(\overline{t_{1} \ldots t_{N}}\right)^{\infty}$ is the quasi-greedy expansion of 1 for some $\beta^{\prime} \in(1,2]$, i.e., $\delta\left(\beta^{\prime}\right)=\left(\overline{t_{1} \ldots t_{N}}\right)^{\infty}$. Since $N$ is the period of the sequence $\delta\left(\beta^{\prime}\right)$, we have $b\left(1, \beta^{\prime}\right)=\overline{t_{1} \ldots t_{N-1}} 10^{\infty}$. So, by Lemma 2.1 (iii) it follows that

$$
\overline{t_{j+1} \ldots t_{N}} \prec \overline{t_{j+1} \ldots t_{N-1}} 1 \preccurlyeq \overline{t_{1} \ldots t_{N-j}}
$$

for all $1 \leq j<N$. Then the lemma follows by taking the reflection in the above equation.

Now we prove the coincidence of the two bifurcation sets.

Lemma 3.4. Let $\beta \in(1,2)$ with $\delta(\beta)=\left(1^{m} 0\right)^{\infty}$. Then $\mathscr{E}_{\beta}=\mathscr{B}_{\beta}$.

Proof. By the definition of the two bifurcation sets it is easy to see that $\mathscr{B}_{\beta} \subset \mathscr{E}_{\beta}$. So in the following we prove $\mathscr{E}_{\beta} \subset \mathscr{B}_{\beta}$.

Let $t \in \mathscr{E}_{\beta}$ with $b(t, \beta)=\left(t_{i}\right)$. Then by Theorem 1.1 we have $t \leq 1-1 / \beta<1 / \beta$. This gives $t_{1}=0$. By Lemmas 2.1 (ii) and Proposition 2.3 (i) it follows that $\sigma^{n}\left(\left(t_{i}\right)\right) \succcurlyeq\left(t_{i}\right)$ for all $n \geq 0$. Let $N \geq 1$ be the smallest index such that $\sigma^{N}\left(\left(t_{i}\right)\right)=\left(t_{i}\right)$. If such an integer $N$ does not exist, then we set $N=\infty$. In the following we will prove $t \in \mathscr{B}_{\beta}$ by considering the following two cases: (I) $N<\infty$; and (II) $N=\infty$.

Case (I). $N<\infty$. We claim that $t_{1} \ldots t_{N}$ is a $\beta$-Lyndon word. If $N=1$, then $\left(t_{i}\right)=t_{1}^{\infty}=$ $0^{\infty}$. It is easy to check that $t_{1}=0$ is a $\beta$-Lyndon word. In the following we assume $N \geq 2$. Since $\sigma^{N}\left(\left(t_{i}\right)\right)=\left(t_{i}\right)$, we have $\left(t_{i}\right)=\left(t_{1} \ldots t_{N}\right)^{\infty}$. Note that $\left(t_{i}\right)$ is the greedy $\beta$-expansion of $t$. Then by Lemma 2.1 (ii) it follows that $\sigma^{n}\left(\left(t_{1} \ldots t_{N}\right)^{\infty}\right) \prec \delta(\beta)$ for all $n \geq 0$. Note that $\sigma^{n}\left(\left(t_{1} \ldots t_{N}\right)^{\infty}\right) \succcurlyeq\left(t_{1} \ldots t_{N}\right)^{\infty}$. Then by Lemma 3.3 and the definition of $N$, it follows that

$$
t_{j+1} \ldots t_{N} \succ t_{1} \ldots t_{N-j} \text { for all } 1 \leq j<N \text {. }
$$

So by Definition 1.2 we establish the claim.

Hence, $t=\left(\left(t_{1} \ldots t_{N}\right)^{\infty}\right)_{\beta}=t_{R}$ is the right endpoint of a $\beta$-Lyndon interval generated by $t_{1} \ldots t_{N}$. By Lemma 3.2 it follows that $\widetilde{K}_{\beta}(t)$ is a transitive subshift of finite type. Observe that for any $t^{\prime}>t$ we have $\widetilde{K}_{\beta}\left(t^{\prime}\right) \subset \widetilde{K}_{\beta}(t)$ and $\left(t_{1} \ldots t_{N}\right)^{\infty} \in \widetilde{K}_{\beta}(t) \backslash \widetilde{K}_{\beta}\left(t^{\prime}\right)$. Recall by [5, Corollary 4.4.9] that for any transitive subshift of finite type, any proper subshift has strictly smaller topological entropy. Therefore,

$$
h\left(\widetilde{K}_{\beta}\left(t^{\prime}\right)\right)<h\left(\widetilde{K}_{\beta}(t)\right) \text { for any } t^{\prime}>t .
$$

By Proposition 2.3 (ii) this yields $\eta_{\beta}\left(t^{\prime}\right)<\eta_{\beta}(t)$ for any $t^{\prime}>t$. So $t \in \mathscr{B}_{\beta}$.

Case (II). $N=\infty$. Then $\sigma^{n}\left(\left(t_{i}\right)\right) \succ\left(t_{i}\right)$ for all $n \geq 1$. So $\left(t_{i}\right)$ is not periodic. Observe that $\left(t_{i}\right)$ begins with digit 0 , and $\sigma^{n}\left(\left(t_{i}\right)\right) \prec\left(1^{m} 0\right)^{\infty}$ for all $n \geq 0$. So there exists a subsequence $\left(m_{k}\right)$ of positive integers such that for any $k \geq 1$ we have $t_{m_{k}}=0$, and the word $t_{1} \ldots t_{m_{k}}^{+}:=$ $t_{1} \ldots t_{m_{k}-1} 1$ does not contain $m+1$ consecutive ones. Then by noting $t_{1}=0$ it follows that

$$
\sigma^{n}\left(\left(t_{1} \ldots t_{m_{k}}^{+}\right)^{\infty}\right) \prec\left(1^{m} 0\right)^{\infty} \quad \forall n \geq 0 .
$$


Since $\sigma^{n}\left(\left(t_{i}\right)\right) \succcurlyeq\left(t_{i}\right)$ for all $n \geq 0$, by Definition 1.2 it follows that $t_{1} \ldots t_{m_{k}}^{+}$is a $\beta$-Lyndon word for any $k \geq 1$. Let $s_{k}:=\left(\left(t_{1} \ldots t_{m_{k}}^{+}\right)^{\infty}\right)_{\beta}$. Then $s_{k}$ is the right endpoint of a $\beta$-Lyndon interval generated by $t_{1} \ldots t_{m_{k}}^{+}$. Furthermore, $s_{k}$ strictly decreases to $t=\left(\left(t_{i}\right)\right)_{\beta}$ as $k \rightarrow \infty$.

So, for any $t^{\prime}>t$ we can find $k$ such that $s_{k} \in\left(t, t^{\prime}\right)$. By the same arguments as in the proof of Case (I) for $s_{k}$ we conclude that

$$
\eta_{\beta}\left(t^{\prime}\right)<\eta_{\beta}\left(s_{k}\right) \leq \eta_{\beta}(t) .
$$

So $t \in \mathscr{B}_{\beta}$. This completes the proof.

Finally, we describe the bifurcation sets via the $\beta$-Lyndon intervals.

Lemma 3.5. Let $\beta \in(1,2]$ with $\delta(\beta)=\left(1^{m} 0\right)^{\infty}$. Then

$$
\left[0,1-\frac{1}{\beta}\right) \backslash \bigcup\left[t_{L}, t_{R}\right) \subset \mathscr{E}_{\beta}
$$

Proof. Take $t \in[0,1-1 / \beta) \backslash \mathscr{E}_{\beta}$ with greedy $\beta$-expansion $\left(t_{i}\right)$. Then $t_{1}=0$. Since $t \notin \mathscr{E}_{\beta}$, by Proposition 2.3 (i) there exists a smallest positive integer $N$ such that $T_{\beta}^{N}(t)<t$, which implies

$$
t_{N+1} t_{N+2} \ldots \prec\left(t_{i}\right) .
$$

We claim that $t_{1} \ldots t_{N}$ is a $\beta$-Lyndon word. Clearly, if $N=1$ then $t_{1}=0$ is a $\beta$-Lyndon word. In the following we assume $N \geq 2$. In view of Definition 1.2 it suffices to prove

$$
t_{j+1} \ldots t_{N} \succ t_{1} \ldots t_{N-j} \text { for all } 1 \leq j<N
$$

and

$$
\sigma^{n}\left(\left(t_{1} \ldots t_{N}\right)^{\infty}\right) \prec\left(1^{m} 0\right)^{\infty} \quad \text { for all } n \geq 0 .
$$

First we prove (3.7). By the definition of $N$ in (3.6) it follows that

$$
t_{j+1} t_{j+2} \ldots \succcurlyeq\left(t_{i}\right) \text { for all } 1 \leq j<N
$$

which implies $t_{j+1} \ldots t_{N} \succcurlyeq t_{1} \ldots t_{N-j}$ for all $1 \leq j<N$. Suppose $t_{j+1} \ldots t_{N}=t_{1} \ldots t_{N-j}$ for some $j \in\{1,2, \ldots, N-1\}$. Then by (3.6) and (3.9) it follows that

$$
t_{j+1} t_{j+2} \ldots=t_{1} \ldots t_{N-j} t_{N+1} t_{N+2} \ldots \prec t_{1} \ldots t_{N-j} t_{1} t_{2} \ldots \preccurlyeq\left(t_{i}\right),
$$

leading to a contradiction with the minimality of $N$. This proves (3.7).

To prove (3.8) we observe that $\delta(\beta)=\left(1^{m} 0\right)^{\infty}$ and $\left(t_{i}\right)$ is the greedy $\beta$-expansion of $t$. Then $t_{1} \ldots t_{N}$ cannot contain $m+1$ consecutive ones. Since $t_{1}=0$, we have $\sigma^{n}\left(\left(t_{1} \ldots t_{N}\right)^{\infty}\right) \preccurlyeq$ $\left(1^{m} 0\right)^{\infty}$ for all $n \geq 0$. So to prove (3.8) it remains to prove that $\sigma^{n}\left(\left(t_{1} \ldots t_{N}\right)^{\infty}\right) \neq\left(1^{m} 0\right)^{\infty}$ for any $n \geq 0$. Suppose the equality $\sigma^{n}\left(\left(t_{1} \ldots t_{N}\right)^{\infty}\right)=\left(1^{m} 0\right)^{\infty}$ holds for some $n \geq 0$. Then using $t_{1}=0$ it follows $t_{1} \ldots t_{m+1}=01^{m}$. This implies $\left(t_{i}\right) \succcurlyeq 01^{m} 0^{\infty}=b(1-1 / \beta, \beta)$. By Lemma 2.1 (ii) we have $t \geq 1-1 / \beta$, leading to a contradiction. So the claim follows. 
By the claim there exists a $\beta$-Lyndon interval $\left[t_{L}, t_{R}\right)$ generated by $t_{1} \ldots t_{N}$. Furthermore, by (3.6) it follows that

$$
\begin{aligned}
\left(t_{i}\right)=t_{1} \ldots t_{N} t_{N+1} t_{N+2} \ldots & \prec t_{1} \ldots t_{N} t_{1} t_{2} \ldots=\left(t_{1} \ldots t_{N}\right)^{2} t_{N+1} t_{N+2} \ldots \\
& \prec\left(t_{1} \ldots t_{N}\right)^{2} t_{1} t_{2} \ldots=\left(t_{1} \ldots t_{N}\right)^{3} t_{N+1} t_{N+2} \ldots \\
& \ldots \\
& \preccurlyeq\left(t_{1} \ldots t_{N}\right)^{\infty} .
\end{aligned}
$$

Therefore, $t_{1} \ldots t_{N} 0^{\infty} \preccurlyeq\left(t_{i}\right) \prec\left(t_{1} \ldots t_{N}\right)^{\infty}$, which gives $t \in\left[t_{L}, t_{R}\right)$ by Lemma 2.1 (ii). This completes the proof.

Proof of Proposition 3.1. By Lemmas 3.4 and 3.5 it suffices to prove

$$
\mathscr{B}_{\beta} \subset\left[0,1-\frac{1}{\beta}\right) \backslash \bigcup\left[t_{L}, t_{R}\right) \text {. }
$$

Note by Lemma 3.4 and Theorem 1.1 that $\mathscr{B}_{\beta}=\mathscr{E}_{\beta} \subset[0,1-1 / \beta]$. In fact we have $\mathscr{E}_{\beta} \subset$ $[0,1-1 / \beta)$. Note that $b(1-1 / \beta, \beta)=01^{m} 0^{\infty}$. So $T_{\beta}^{m+1}(1-1 / \beta)<1-1 / \beta$. By Proposition 2.3 (i) this implies $1-1 / \beta \notin \mathscr{E}_{\beta}$. Hence, $\mathscr{E}_{\beta} \subset[0,1-1 / \beta)$.

In the following it remains to prove $\mathscr{B}_{\beta} \cap \bigcup\left[t_{L}, t_{R}\right)=\emptyset$. If $t \in\left[t_{L}, t_{R}\right)$, then by (3.1) it follows that $\widetilde{K}_{\beta}(t)=\widetilde{K}_{\beta}\left(t_{L}\right)=\widetilde{K}_{\beta}\left(t_{R}\right)$, which gives $\eta_{\beta}\left(t^{\prime}\right)=\eta_{\beta}(t)=\eta_{\beta}\left(t_{L}\right)$ for all $t^{\prime} \in\left(t, t_{R}\right)$. So, $t \notin \mathscr{B}_{\beta}$. This completes the proof.

As a consequence of Proposition 3.1 and Theorem 1.1 it follows that for $\beta \in(1,2]$ a multinacci number the $\beta$-Lyndon intervals cover $[0,1-1 / \beta)$ up to a Lebesgue null set.

Corollary 3.6. Let $\beta \in(1,2]$ be a multinacci number.

(i) The union of all $\beta$-Lyndon intervals covers $[0,1-1 / \beta)$ up to a Lebesgue null set. Furthermore, for any $t \in \mathscr{B}_{\beta}$ and any $r>0$ the interval $(t, t+r)$ contains infinitely many $\beta$-Lyndon intervals.

(ii) $\eta_{\beta}(t)>0$ if and only if $t<1-1 / \beta$.

Proof. Note by Theorem 1.1 that $\mathscr{E}_{\beta}$ is a Lebesgue null set with no isolated points. Then (i) follows from Proposition 3.1 which tells us that $\bigcup\left[t_{L}, t_{R}\right)=[0,1-1 / \beta) \backslash \mathscr{E}_{\beta}$. For (ii) it can be deduced from Proposition 3.1 and Theorem 1.1 that $\sup \mathscr{B}_{\beta}=1-1 / \beta$ and $1-1 / \beta \notin \mathscr{B}_{\beta}$.

Now we turn to investigate the local dimension of the bifurcation set $\mathscr{B}_{\beta}$.

Lemma 3.7. Let $\beta \in(1,2]$ with $\delta(\beta)=\left(1^{m} 0\right)^{\infty}$. Then

$$
\lim _{r \rightarrow 0} \operatorname{dim}_{H}\left(\mathscr{B}_{\beta} \cap(t, t+r)\right)=\operatorname{dim}_{H} K_{\beta}(t)>0 \quad \forall t \in \mathscr{B}_{\beta} .
$$

Proof. Take $t \in \mathscr{B}_{\beta}$. By Proposition 3.1 we have $t<1-1 / \beta$, and then by Corollary 3.6 (ii) it gives $\eta_{\beta}(t)=\operatorname{dim}_{H} K_{\beta}(t)>0$. Note by Proposition 3.1 and Proposition 2.3 (i) that $\mathscr{B}_{\beta} \cap(t, t+r)=\mathscr{E}_{\beta} \cap(t, t+r) \subseteq K_{\beta}(t)$ for any $r>0$. Then $\lim _{r \rightarrow 0} \operatorname{dim}_{H}\left(\mathscr{B}_{\beta} \cap(t, t+r)\right) \leq \eta_{\beta}(t)$. So it remains to prove

$$
\lim _{r \rightarrow 0} \operatorname{dim}_{H}\left(\mathscr{B}_{\beta} \cap(t, t+r)\right) \geq \eta_{\beta}(t) .
$$

We prove this now by considering the following two cases: (I) $t=t_{R}$ is the right endpoint of a $\beta$-Lyndon interval; (II) $t \in[0,1-1 / \beta) \backslash \bigcup\left[t_{L}, t_{R}\right]$. 
Case (I). Suppose $t=t_{R}$ is the right endpoint of a $\beta$-Lyndon interval. Let $\left(t_{i}\right)=\left(t_{1} \ldots t_{p}\right)^{\infty}$ be the greedy $\beta$-expansion of $t_{R}$. Note that $t_{R} \in \mathscr{B}_{\beta}$. Then by Corollary [3.6 (i) there exists a sequence $\left(t_{R}^{(n)}\right) \subset \mathscr{B}_{\beta}$ such that each $t_{R}^{(n)}$ is a right endpoint of a $\beta$-Lyndon interval and $t_{R}^{(n)} \searrow t_{R}$ as $n \rightarrow \infty$. Fix $r>0$. There exists a large integer $N$ satisfying $t_{R}^{(n)} \in\left(t_{R}, t_{R}+r\right)$ for all $n \geq N$. Furthermore, by Lemma 2.1 (ii) it follows that for each $n \geq N$ there exists an integer $k_{n}$ such that the greedy $\beta$-expansion $b\left(t_{R}^{(n)}, \beta\right)$ of $t_{R}^{(n)}$ satisfies

$$
b\left(t_{R}^{(n)}, \beta\right) \succ\left(t_{1} \ldots t_{p}\right)^{k_{n}} 1^{\infty} .
$$

Observe by Proposition 3.1 and Proposition 2.3 (i) that

$$
\mathscr{B}_{\beta}=\mathscr{E}_{\beta}=\left\{\left(\left(s_{i}\right)\right)_{\beta}:\left(s_{i}\right) \preccurlyeq \sigma^{n}\left(\left(s_{i}\right)\right) \prec\left(1^{m} 0\right)^{\infty} \forall n \geq 0\right\} .
$$

So by using $t_{R} \in \mathscr{B}_{\beta}$, (3.11) and Lemma 2.1 (ii) it follows that for any $n \geq N$,

$$
\begin{aligned}
\left\{\left(\left(t_{1} \ldots t_{p}\right)^{k_{n}} x_{1} x_{2} \ldots\right)_{\beta}: x_{1} \ldots x_{p}=t_{1} \ldots t_{p},\left(x_{i}\right) \in \widetilde{K}_{\beta}\left(t_{R}^{(n)}\right)\right\} & \subseteq \mathscr{B}_{\beta} \cap\left[t_{R}, t_{R}^{(n)}\right) \\
& \subseteq \mathscr{B}_{\beta} \cap\left[t_{R}, t_{R}+r\right) .
\end{aligned}
$$

Note by Lemma 3.2 that $\widetilde{K}_{\beta}\left(t_{R}^{(n)}\right)$ is a transitive subshift of finite type. Then by (3.12) it follows that

$$
\operatorname{dim}_{H}\left(\mathscr{B}_{\beta} \cap\left(t_{R}, t_{R}+r\right)\right) \geq \operatorname{dim}_{H} K_{\beta}\left(t_{R}^{(n)}\right)=\eta_{\beta}\left(t_{R}^{(n)}\right) \quad \text { for all } n \geq N .
$$

Letting $n \rightarrow \infty$ and by the continuity of $\eta_{\beta}$ we obtain that

$$
\operatorname{dim}_{H}\left(\mathscr{B}_{\beta} \cap\left(t_{R}, t_{R}+r\right)\right) \geq \eta_{\beta}\left(t_{R}\right) .
$$

Since $r>0$ was given arbitrary, letting $r \rightarrow 0$ we conclude that

$$
\lim _{r \rightarrow 0} \operatorname{dim}_{H}\left(\mathscr{B}_{\beta} \cap\left(t_{R}, t_{R}+r\right)\right) \geq \eta_{\beta}\left(t_{R}\right) .
$$

Case (II). $t \in\left[0,1-\frac{1}{\beta}\right) \backslash \bigcup\left[t_{L}, t_{R}\right]$. Then by Corollary 3.6 (i) there exists a sequence $\left(t_{R}^{(k)}\right)$ such that each $t_{R}^{(k)}$ is the right endpoint of a $\beta$-Lyndon interval, and $t_{R}^{(k)} \searrow t$ as $k \rightarrow \infty$. So, for any $r>0$ there exists a sufficiently large integer $k$ such that $t_{R}^{(k)} \in(t, t+r)$. By (3.13) with $t_{R}$ replaced by $t_{R}^{(k)}$ it follows that for any $\varepsilon>0$ there exists $r_{k}>0$ such that $\left(t_{R}^{(k)}, t_{R}^{(k)}+r_{k}\right) \subset(t, t+r)$ and then

$$
\operatorname{dim}_{H}\left(\mathscr{B}_{\beta} \cap(t, t+r)\right) \geq \operatorname{dim}_{H}\left(\mathscr{B}_{\beta} \cap\left(t_{R}^{(k)}, t_{R}^{(k)}+r_{k}\right)\right) \geq \eta_{\beta}\left(t_{R}^{(k)}\right)-\varepsilon .
$$

Letting $r \rightarrow 0$, and then $t_{R}^{(k)} \rightarrow t$, we conclude by the continuity of $\eta_{\beta}$ that

$$
\lim _{r \rightarrow 0} \operatorname{dim}_{H}\left(\mathscr{B}_{\beta} \cap(t, t+r)\right) \geq \eta_{\beta}(t)-\varepsilon .
$$

Since $\varepsilon>0$ was arbitrary, we obtain $\lim _{r \rightarrow 0} \operatorname{dim}_{H}\left(\mathscr{B}_{\beta} \cap(t, t+r)\right) \geq \eta_{\beta}(t)$. This, together with (3.13), proves (3.10).

Proof of Theorem 1. Let $\beta \in(1,2)$ with $\delta(\beta)=\left(1^{m} 0\right)^{\infty}$. By Lemma 2.2. Proposition 3.1 and Lemma 3.7 it suffices to prove

$$
\left\{t \in[0,1): \lim _{r \rightarrow 0} \operatorname{dim}_{H}\left(\mathscr{B}_{\beta} \cap(t, t+r)\right)=\eta_{\beta}(t)>0\right\} \subset \mathscr{B}_{\beta} .
$$


Take $t \in[0,1) \backslash \mathscr{B}_{\beta}$. Then by Proposition 3.1 we have $t \in[1-1 / \beta, 1)$ or $t \in\left[t_{L}, t_{R}\right)$ for some $\beta$-Lyndon interval. If $t \geq 1-1 / \beta$, then $\eta_{\beta}(t)=0$ by Corollary [3.6 (ii). If $t \in\left[t_{L}, t_{R}\right)$, then there exists $r>0$ such that $\mathscr{B}_{\beta} \cap(t, t+r)=\emptyset$. This completes the proof.

Proof of Corollary 2. Note by Proposition 3.1 that $\mathscr{E}_{\beta} \subset[0,1-1 / \beta)$. So if $t \geq 1-1 / \beta$, then clearly the result holds by Corollary 3.6 (ii). Now let $t \in[0,1-1 / \beta)$. Observe by Proposition 2.3 (i) that $\mathscr{E}_{\beta} \cap[t, 1] \subset K_{\beta}(t)$. So it suffices to prove

$$
\operatorname{dim}_{H}\left(\mathscr{E}_{\beta} \cap[t, 1]\right) \geq \operatorname{dim}_{H} K_{\beta}(t) .
$$

If $t \in[0,1-1 / \beta) \backslash\left[t_{L}, t_{R}\right)$, then (3.15) follows by Lemma 3.7, If $t \in\left[t_{L}, t_{R}\right)$, then we still have (3.15) by using Lemma 3.7 that

$$
\operatorname{dim}_{H}\left(\mathscr{E}_{\beta} \cap[t, 1]\right) \geq \operatorname{dim}_{H}\left(\mathscr{E}_{\beta} \cap\left[t_{R}, 1\right]\right) \geq \operatorname{dim}_{H} K_{\beta}\left(t_{R}\right)=\operatorname{dim}_{H} K_{\beta}(t),
$$

where the last equality holds by (3.1).

\section{Final Remarks}

The main results obtained in this paper can be easily modified to study the following analogous bifurcation sets:

$$
\begin{aligned}
& \mathscr{E}_{\beta}^{\prime}:=\left\{t \in[0,1): K_{\beta}\left(t^{\prime}\right) \neq K_{\beta}(t) \forall t^{\prime} \neq t\right\}, \\
& \mathscr{B}_{\beta}^{\prime}:=\left\{t \in[0,1): \operatorname{dim}_{H} K_{\beta}\left(t^{\prime}\right) \neq \operatorname{dim}_{H} K_{\beta}(t) \forall t^{\prime} \neq t\right\} .
\end{aligned}
$$

If $\beta \in(1,2]$ is a multinacci number, one can show that

$$
\begin{aligned}
\mathscr{B}_{\beta}^{\prime} & =\mathscr{E}_{\beta}^{\prime}=\left[0,1-\frac{1}{\beta}\right) \backslash \bigcup\left[t_{L}, t_{R}\right] \\
& =\left\{t \in[0,1): \lim _{r \rightarrow 0} \operatorname{dim}_{H}\left(\mathscr{E}_{\beta} \cap(t-r, t)\right)=\lim _{r \rightarrow 0} \operatorname{dim}_{H}\left(\mathscr{E}_{\beta} \cap(t, t+r)\right)=\operatorname{dim}_{H} K_{\beta}(t)>0\right\},
\end{aligned}
$$

where the union is taken over all pairwise disjoint closed $\beta$-Lyndon intervals.

Observe that the main result Theorem 1 holds under the assumption that $\beta \in(1,2]$ is a multinacci number, i.e., $\delta(\beta)=\left(1^{m} 0\right)^{\infty}$ for some $m \in \mathbb{N}$. The method used in this paper can be adapted to show that Theorem 1 still holds for $\beta \in(1,2]$ with $\delta(\beta)=\left(10^{m}\right)^{\infty}$. It is worth mentioning that in [4] Kalle et al. considered a general Farey word base $\beta$, i.e., $\delta(\beta)=\left(s_{1} \ldots s_{p}\right)^{\infty}$ with $s_{m} s_{m-1} \ldots s_{2} s_{1}$ a non-degenerate Farey word. They showed that for a general Farey word base $\beta \in(1,2)$, the set-valued bifurcation set $\mathscr{E}_{\beta}$ has no isolated points and Theorem 1.1 holds. We finish by posing the following conjecture.

Conjecture 4.1. Let $\beta \in(1,2]$. Then $\mathscr{B}_{\beta}=\mathscr{E}_{\beta}$ if and only if $\mathscr{E}_{\beta}$ has no isolated points.

\section{ACKNOWLEDGEMENTS}

The authors were supported by an LMS Scheme 4 grant. The first author was supported by EPSRC grant EP/M001903/1. The second author was supported by NSFC No. 11401516, and by the Fundamental Research Funds for the Central Universities No. 2019CDXYST0015. He wishes to thank the Mathematical Institute of Leiden University. 


\section{REFERENCES}

[1] C. Carminati and G. Tiozzo. The local Hölder exponent for the dimension of invariant subsets of the circle. Ergodic Theory Dynam. Systems, 37(6):1825-1840, 2017.

[2] Z. Daróczy and I. Kátai. Univoque sequences. Publ. Math. Debrecen, 42(3-4):397-407, 1993.

[3] M. de Vries and V. Komornik. Unique expansions of real numbers. Adv. Math., 221(2):390-427, 2009.

[4] C. Kalle, D. Kong, N. Langeveld, and W. Li. The $\beta$-transformation with a hole at 0. arXiv:1803.07338. To appear in Ergodic Theory Dynam. Systems.

[5] D. Lind and B. Marcus. An introduction to symbolic dynamics and coding. Cambridge University Press, Cambridge, 1995.

[6] R. D. Mauldin and S. C. Williams. Hausdorff dimension in graph directed constructions. Trans. Amer. Math. Soc., 309(2):811-829, 1988.

[7] W. Parry. On the $\beta$-expansions of real numbers. Acta Math. Acad. Sci. Hungar., 11:401-416, 1960.

[8] P. Raith. Hausdorff dimension for piecewise monotonic maps. Studia Math., 94(1):17-33, 1989.

[9] A. Rényi. Representations for real numbers and their ergodic properties. Acta Math. Acad. Sci. Hungar., 8:477-493, 1957.

[10] N. Sidorov. Almost every number has a continuum of $\beta$-expansions. Amer. Math. Monthly, 110(9):838-842, 2003.

[11] M. Urbański. On Hausdorff dimension of invariant sets for expanding maps of a circle. Ergodic Theory Dynam. Systems, 6(2):295-309, 1986.

[12] M. Urbański. Invariant subsets of expanding mappings of the circle. Ergodic Theory Dynam. Systems, 7(4):627-645, 1987.

(S. Baker) Mathematics institute, University of Warwick, Coventry, CV4 7AL, UK

E-mail address: simonbaker412@gmail.com

(D. Kong) College of Mathematics And Statistics, Chongqing University, 401331, Chongqing, P.R.CHINA

E-mail address: derongkong@126.com 